\title{
Über den fermentativen Abbau der Hemicellulosen.
}

\section{Mitteilung.}

Ein Trisaccharid als Zwischenprodukt der Hydrolyse eines Mannans.

Von

Hans Pringsheim.

(Aus dem chemischen Institut der Universität Berlin.)

(Der Redaktion zugegangen am 5. August 1912.)

Der hydrolytische Abbau der Hemicellulosen ist schon des öfteren beschrieben worden, speziell liegen auch über die Mannane spaltenden Fermente (Seminasen, Carobinasen) verschiedene Untersuchungen vor. ${ }^{1}$ ) Bei diesen Abbauversuchen wurden jedoch stets Monosaccharide als Endprodukte der fermentativen Hydrolyse gewonnen, ja Bierry und Giaja ${ }^{2}$ ) haben vor kurzem angegeben, da $\beta$ sie bei diesem Abbau vergeblich nach einem Disaccharid gefahndet haben. In dieser Beziehung bin ich glücklicher gewesen. Als ich die Methode, welche ich für den fermentativen Abbau der echten Cellulose angegeben habe ${ }^{3}$ ) auf das in den Steinnußspänen vorhandene Mannan anwandte, gelang es mir, durch Isolierung der Osazone zu zeigen, daß hierbei neben dem Endprodukte der Hydrolyse, der Mannose, ein Trisaccharid als Zwischenprodukt gebildet wird.

Die Steinnußspäne sind für einen derartigen Versuch besonders geeignet, da sie sehr reich an Mannose sind, bilden sie doch das hauptsächlichste Ausgangsmaterial zur Darstellung dieses Monosaccharids. Trotz alledem ist der Beweis noch nicht

1) Grüß, Ber. d. deutsch. bot. Gesellsch., Bd. 12, S. 60 (1894); Bourquelot und Hérissey, Compt. rend. de l'Académie, Vol. 130, p. 1719 (1900), Vol. 131, p. 113, 903 (1901), Vol. 136, p. 1193, 1404 (1903). Hérissey, ebenda, Vol. 134, p. 721 (1902). Weitere Literatur: Biochemisches Handlexikon, Bd. 2, S. 50/51.

S. 370 (1912).

$\left.{ }^{2}\right)$ H. Bierry und J. Giaja, Biochemische Zeitschrift, Bd. 40,

3) Hans Pringsheim, Diese Zeitschrift, Bd. 78, S. 266 (1912). 
erbracht, daß die Mannose der einzige zuckerartige Konstituent des Mannans der Steinnußspäne ist; denn da sie über ihr schwerlösliches Phenylhydrazon gewonnen wird, so ist nicht sicher zu erschließen, ob in der Hydrolyseflüssigkeit der Späne nicht noch andere Zucker vorhanden sind, die mit Phenylhydrazin in der Kälte keine schwerlösliche Verbindung geben. Immerhin ist es sehr wahrscheinlich, daß wir es hier mit einem reinen Mannan zu tun haben. Entfernt man nämlich aus dem Säurehydrolysat der Steinnußspäne die Mannose durch Ausfällen mit Phenylhydrazin, womit sie ja quantitativ gefällt werden kann, ${ }^{1}$ ) so wird beim Erwärmen mit einem Überschuß von Phenylhydrazin kein Osazon eines Monosaccharids mehr gebildet. Das müßte aber der Fall sein, wenn noch andere Monosaccharide als Mannose, z. B. Glukose, Fruktose, Galaktose oder auch Arabinose usw. vorhanden wären. Man konnte weiter daran denken, durch den Vergleich der Drehung und der Reduktionskraft gegenüber Fehlingscher Lösung den Beweis für das ausschließliche Vorhandensein von Mannose zu erbringen. Solche Versuche scheitern aber, offenbar an der Anwesenheit noch anderer Stoffe nicht zuckerartiger Natur, welche diese Konstanten beeinflussen. - So muß man sich mit der sehr wahrscheinlichen Vermutung begnügen, daß es sich bei dem Zwischenprodukt des Abbaus um eine Trimannose handelt, solange es nicht gelingt, diesen Zucker krystallinisch abzuscheiden. Auf jeden Fall ist die Tatsache bemerkenswert, daß in diesem fermentativen Abbau ein Trisaccharid entsteht, da bisher nur Disaccharide, wie bei der Cellulose, und noch nie Trisaccharide als fermentative Abbauprodukte aufgefunden worden sind.

Auch ohne daß das Trisaccharid in Substanz abgeschieden wurde, läßt sich über das Verhalten dieses Zuckers einiges aussagen. Er wird von gewöhnlicher Preßhefe komplett ver-

1) Fischer und Hirschberger, Ber. d. deutsch. chem. Gesellsch., Jg. 21, S. 1805 (1888); Bourquelot und Hérissey, Compt. rend. de l'Acad., Vol. 129, p. 339 (1899).

2) H. Ghr. Geelmuyden, Zeitschr. f. analytische Chemie, Bd. 48, S. 137 (1909). 
goren. Ein Weg, um die bei dem fermentativen Abbau immer neben ihm auftretende Mannose wegzunehmen, wurde in der Nichtvergärbarkeit des Trisaccharids durch die Hefe Nr. 583 der Versuchs- und Lehranstalt für Brauerei in Berlin, die auch Maltose unvergoren läßt, aufgefunden. Auf diese Weise gelingt es, das Trisaccharid als einzigen Zucker in der Verdauungsflüssigkeit zurückzubehalten. Mit dieser Lösung konnte dann auch der Beweis erbracht werden, daß das Trisaccharid durch Emulsin gespalten wird, und daß hierbei neben einem Monosaccharid aller Wahrscheinlichkeit nach ein Disaccharid entsteht. Dies würde auch dem Verhalten seines Osons gegen Emulsin entsprechen. Jedoch erfolgt, wie zu erwarten war, die Spaltung durch Emulsin nicht komplett; die Folge davon war, daß das Osazon des Disaccharids, als welches es isoliert wurde, noch mit dem des Trisaccharids gemengt, auskrystallisierte. Auf diesem Wege kann also keine Lösung des reinen Disaccharids gewonnen werden. Ich glaube aber, daß ich durch Vergärung des Trisaccharids mit Obergärhefe zu einem Disaccharid gelangen kann, da einzelne Versuche für ein solches Verhalten sprechen. Dieses Disaccharid mülite dann aber, entsprechend dem Verhalten der Raffinose gegen Emulsin und Obergärhefe, von dem bei der Emulsinspaltung erzeugten verschieden sein.

\section{Experimenteller Teil.}

Die Bakterienkultur zur Vergärung der Steinnußspäne wurde durch Beimpfen der Aufschwemmung des Mannans in Leitungswasser, mit schwefelsaurem Ammoniak als Stickstoffquelle, in Gegenwart von kohlensaurem Kalk (zur Abstumpfung der sich bildenden Säuren) mit Erde erhalten. Nach der Inkubation bei $37^{\circ} \mathrm{C}$. setzte schon in wenigen Tagen eine Gärung ein, die weiter nicht bakteriell untersucht wurde. Sie diente als Impfflüssigkeit und konnte monatelang zur Einleitung neuer Gärungen benutzt werden.

$250 \mathrm{~g}$ Steinnußspäne, $50 \mathrm{~g}\left(\mathrm{NH}_{4}\right)_{2} \mathrm{SO}_{4}$ und $20 \mathrm{~g} \mathrm{CaCO}_{3}$ wurden in $6 \mathrm{l}$ Wasser aufgeschwemmt, drei Tage lang der Gärung überlassen, die unter ziemlich starker Gasabgabe vor 
sich ging. Dann wurde die Gärung durch kräftiges Schülteln mit $5 \mathrm{ccm}$ Toluol angehalten, woraufhin sich in weiteren 2 bis 3 Tagen deutliche Reduktion der Verdauungsflüssigkeit gegen Fehlingsche Lösung bemerkbar machte. Jetzt wurde durch Filtration von den Steinnußspänen getrennt, die nach dem Waschen mit Wasser unter Zusatz von etwas Alkohol immer zu neuen Abbauversuchen verwandt werden konnten. Das Filtrat wurde auf $100 \mathrm{~cm}$ eingedampft, mit Alkohol gefällt, vom ausfallenden Niederschlag abfiltriert und die alkoholische Flüssigkeit im Vakuum zur Trockne verdampft. Dann wurde mit $50 \mathrm{ccm}$ Wasser aufgenommen und $25 \mathrm{ccm}$ der Lösung mit Preßhefe vergoren. Nach 48 Stunden bei $25^{\circ}$ war keine Reduktion gegen Fehlingsche Lösung und keine Osazonbildung mehr zu beobachten. Die andern $25 \mathrm{ccm}$ wurden in der Kälte

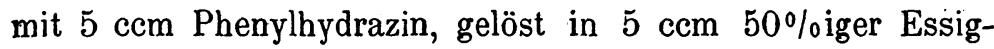
säure, versetzt. Hierbei fiel nach 24 stündigem Stehen nur eine geringe Menge von Mannosephenylhydrazon, da seine völlige Ausfällung offenbar durch die Anwesenheit des Trisaccharids verhindert worden war. In mehreren Versuchen wurde hierbei dasselbe Resultat erhalten. Das Mannosephenylhydrazon zeigte nach dem Umkrystallisieren aus kochendem Wasser den Zersetzungspunkt von 196-1970 (korr.).

Ein weiterer Versuch wurde mit 21 der Verdauungsflüssigkeit unternommen. Es wurde von jetzt an aber nicht mehr mit Alkohol, sondern mit Soda in der Kälte gefällt, wobei die Kalksalze der bei der Vergärung gebildeten Säuren zerlegt werden. Nach der Filtration des kohlensauren Kalks wurde schwach mit Essigsäure angesäuert und nach Zusatz von $5 \mathrm{ccm}$ Phenylhydrazin, gelöst in $5 \mathrm{ccm} 50 \%$ iger Essigsäure, zwei Stunden im kochenden Wasserbade erhitzt. Nach dem Erkalten wurde das Gemisch der gebildeten Osazone abfiltriert, zuerst mit kaltem Wasser und dann mit Chloroform gewaschen. Durch Auskochen mit $50 \mathrm{ccm}$ Wasser wurde darauf das lösliche Osazon vom Glukosazon getrennt. Es krystallisierte aus dem Wasser in schönen, zu Sternen angeordneten Nadeln und war nach nochmaligem Umkrystallisieren aus Wasser analysenrein. Sein Zersetzungspunkt lag bei $196^{\circ}$ C. (korr.). Nach dem Lösen in 
Aceton und nochmaligem Ausfällen mit Petroläther war er ungeằndert.

0,2271 g Substanz: $0,4380 \mathrm{~g} \mathrm{CO}_{2}, 0,1306 \mathrm{~g} \mathrm{H}_{2} \mathrm{O}-0,3698 \mathrm{~g}$ Substanz gaben $25,8 \mathrm{~cm} \mathrm{~N}$ bei $19^{\circ}$ und $768 \mathrm{~mm}$.

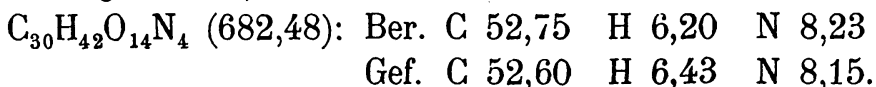

Erhalten wurden $0,5 \mathrm{~g}$ des Triosazons und $0,35 \mathrm{~g}$ des einmal aus verdünntem Alkohol umkrystallisierten Glukosazons vom Zersetzungspunkt $207^{\circ}$.

$0,1 \mathrm{~g}$ des Osazons des Trisaccharids drehten in einem Gemisch von $2 \mathrm{ccm}$ Pyridin und $3 \mathrm{ccm}$ absolutem Alkohol bei Auerlicht $0,21^{\circ} \pm 0,02^{\circ}$ nach links.

Trennung des Trisaccharids von dem Monosaccharid.

1/2 1 sterile Bierwürze wurden mit einer Reinkultur der Kahmhefe Nr. 583 beimpft. Nachdem sich die Hefe zwei Tage lang bei $23^{\circ}$ entwickelt hatte, wurde die überstehende Würze vom Bodensatz der Hefe abgegossen. Zu dieser Hefe goß man den Eindampfrückstand aus 31 Verdauungsflüssigkeit in $50 \mathrm{ccm}$ Wasser. Dann wurde 24 Stunden bei derselben Temperatur der Gärung überlassen, die Hefe über einem mit ein wenig Kieselgur bedeckten Filter abgesaugt; das Filtrat mit Soda gefällt und das Filtrat des hierbei ausfallenden Niederschlages mit einer Lösung von $3 \mathrm{~g}$ Phenylhydrazin in $6 \mathrm{ccm} 50 \%$ iger Essigsäure versetzt. Nach 2 Stunden langem Erhitzen im kochenden Wasserbade wurde keine Spur des schwer löslichen Osazons gefällt, ein Beweis, daß alles Monosaccharid vergoren war. Beim Erkalten fiel das lösliche Osazon in schönen Krystallen aus, die nach dem Waschen mit Wasser und Chloroform, und nach nochmaligem Umkrystallisieren aus wenig Wasser als das Osazon des Trisaccharids identifiziert werden konnten. Ausbeute 0,6 g.

$0,3114 \mathrm{~g}$ Subst. gaben $22,9 \mathrm{ccm} \mathrm{N}$ bei $21^{\circ}$ und $763 \mathrm{~mm}$.

$$
\begin{array}{r}
\mathrm{C}_{30} \mathrm{H}_{42} \mathrm{O}_{14} \mathrm{~N}_{4}(682,48) \text { Ber.: } \mathrm{N} 8,23 \\
\text { Gef.: } \mathrm{N} 8,21 .
\end{array}
$$

Es war also durch die Vergärung mit der Hefe 583 gelungen, eine Lösung des Trisaccharids frei von andern Zuckern 
zu erhalten und man kann hoffen, aus dieser Lösung den Zucker noch kristallinisch zu gewinnen.

Spaltungen mit Emulsin.

0,6 $\mathrm{g}$ des Osazons des Trisaccharids wurden in sein Oson verwandelt. ${ }^{1}$ ) Das Oson wurde in $20 \mathrm{ccm}$ Wasser gelöst mit $0,7 \mathrm{~g}$ Emulsin (Kahlbaum), welches vorher in seiner Wirksamkeit gegen Milchzucker geprüft war, bei Gegenwart von Toluol 3 Tage lang bei $37^{\circ}$ auf bewahrt. Dann wurde vom Emulsin abfiltriert, mit $1 \mathrm{~g}$ Natriumacetat und einem Tropfen Essigsäure aufgekocht und der ausfallende Niederschlag wiederum durch Filtration abgetrennt. Die mit $0,7 \mathrm{~g}$ Phenylhydrazinchlorhydrat versetzte Lösung wurde nun 2 Stunden im kochenden Wasserbade erhitzt. Es fiel schwerlösliches Osazon aus, das nach dem Umkrystallisieren aus verdünntem Alkohol als Glukosazon identifiziert werden konnte. Ausbeute $0,1 \mathrm{~g}$. Zersetzungspunkt $207^{\circ}$. Der ungespaltene Teil des Osons hätte leichtlösliches Osazon ergeben müssen. Das war in der Tat der Fall, doch war seine Menge zu gering, um es zu reinigen und zur Analyse zu bringen. Es war also durch den Versuch nur bewiesen, daß das Oson des Trisaccharids durch Emulsin gespalten worden war. Dagegen war noch unentschieden, ob die Spaltung nur in das Monosaccharid oder in Monosaccharid und Disaccharid erfolgt war. Darüber gibt der nächste Versuch Auskunft.

Der aus 31 Verdauungsflüssigkeit stammende Eindampfrückstand wurde zuerst wieder mit Hefe 583 vergoren. Dann wurde in einem kleinen Teil durch Behandeln mit Phenylhydrazin geprüft, ob alles Monosaccharid vergoren war. Nachdem das bewiesen worden war, wurde der Hauptanteil in $50 \mathrm{ccm}$ Wasser mit 0,7 g Emulsin bei Gegenwart von Toluol drei Tage lang bei $37^{\circ}$ aufbewahrt. Darauf wurde vom Emulsin abfiltriert, mit $3 \mathrm{~g}$ Natriumacetat und einem Tropfen Essigsäure aufgekocht und von neuem filtriert. Die mit $\mathbf{2}$ g Phenylhydrazinchlorhydrat versetzte Lösung ergab nach 2 stündigem Erhitzen im Wasser-

1) E. Fischer und E. F. Armstrong. Ber. d. deutsch. chem. Gesellsch., Jg. 35, S. 3147 (1902). 
bade ein Gemisch von Osazonen, die wiederum durch heißes Wasser in das schwerlösliche Glukosazon $(0,12 \mathrm{~g}$ rom Zersetzungspunkt $207^{\circ}$ nach dem Umkrystallisieren aus verdünntem Alkohol) und das leicht lösliche Osazon getrennt werden konnten.

Letzteres analysierte nach nochmaliger Krystallisation aus heißem Wasser, wobei es in schönen Nadeln ausfiel.

$0,1334 \mathrm{~g}$ gaben $11.2 \mathrm{ccm} \mathrm{N}$ bei $20^{\circ}$ und $762 \mathrm{~mm}$. Gef. : $9,07 \% \mathrm{~N}$.

Die Stickstoffbestimmung zeigte also, daß ein Gemisch der Osazone des Trisaccharids $(8,23 \% \mathrm{~N})$ und des Disaccharids $(10,77 \% \mathrm{~N})$ vorgelegen hatte. Eine komplette Spaltung des Trisaccharids war von vornherein nicht $\mathrm{zu}$ erwarten. Wäre die Spaltung aber so erfolgt, daß das Trisaccharid in drei Moleküle Monosaccharid unter dem Einflusse des Emulsins erfolgt wäre, so hätte das lösliche Osazon auf das Triosazon stimmende Werte geben müssen. Die teilweise Spaltung in ein Molekül Monosaccharid und ein Molekül Disaccharid ist demnach bewiesen.

In einem weiteren Versuch wurde der Verdauungsrückstand mit Obergärhefe vergoren. Hierbei war wieder alles Monosaccharid verschwunden. Das Osazon des zurückbleibenden Zuckers analysierte jedoch für ein Osazon des Disaccharids noch etwas zu niedrig. $(9,52 \% \mathrm{~N})$. Der Versuch muß also wiederholt werden.

Die Deutsche Landwirtschaftsgesellschaft hat mich in der Ausführung dieser Versuche wieder durch die Bewilligung von Mitteln unterstützt. Ihr, wie Herrn Dr. A. Langhans, der mir mit Geschick zur Seite stand, bin ich zu Dank verpflichtet.

Charlottenburg, den 3. Juli 1912. 